Article

\title{
Preparation and Molecular Dynamics Simulation of RDX/MUF Nanocomposite Energetic Microspheres with Reduced Sensitivity
}

\author{
Xinlei Jia ${ }^{1, *}$, Yingying $\mathrm{Hu}^{1}$, Lanjuan $\mathrm{Xu}^{1}{ }^{1}$, Xuewen Liu ${ }^{1}$, Youying $\mathrm{Ma}^{1}{ }^{1}$, Mingming Fu ${ }^{1}$, \\ Jingyu Wang ${ }^{2, *}$ and Jing $\mathrm{Xu}^{3}$ \\ 1 College of Chemical Engineering and Safety, Binzhou University, Binzhou 256600, China \\ 2 School of Enviroment and Safety Engineering, North University of China, Taiyuan 030051, China \\ 3 Administrative Examination and Approval Authority, Binzhou 256600, China \\ * Correspondence: jiaxinleibzu@mail.sdu.edu.cn (X.J.); wjywjy67@163.com (J.W.)
}

Received: 16 July 2019; Accepted: 30 September 2019; Published: 2 October 2019

\begin{abstract}
In order to improve the general problem of irregular coating morphology and low mechanical strength of the coating layer in existing coating desensitization technology, nano-cyclotrimethylene trinitramine/melamine-urea-formaldehyde (RDX/MUF) composite energetic microspheres were prepared by an improved emulsion polymerization, taking the MUF as the binder and RDX as the main explosive. In order to judge whether RDX/MUF possessed good stability, the combination of differential scanning calorimetry (DSC) and molecular dynamics (MD) simulation was used to determine the level of binding binding energy between urea-formaldehyde resin binder (UF) and RDX. In addition, to investigate the optimal reaction temperature for the preparation of MUF/RDX, the binding energy between UF and RDX at different temperatures was simulated. And then the morphology and thermal properties of the as-prepared composite energetic microspheres were analyzed by scanning electron microscopy (SEM) and DSC, the impact sensitivity and friction sensitivity of the resultant samples were tested as well. Moreover, RDX/MUF with the same MUF content was prepared by physical mixing for comparative analysis. MD simulation demonstrated that UF and RDX possessed good binding ability at $298 \mathrm{~K}$. The DSC method indicatec that UF and RDX had good compatibility, and the comprehensive performance of RDX after coating was not significantly deteriorated; The optimal binding temperature between UF and RDX was $60 \sim 70{ }^{\circ} \mathrm{C}$ which is consistent with the experimental results. The experimental results showed that the optimum process conditions for the preparation of RDX/MUF could be listed as follows: the temperature for preparing RDX/MUF composite energetic microspheres by the improved emulsion polymerization was $70{ }^{\circ} \mathrm{C}$ the optimal $\mathrm{pH}$ value of the urea-formaldehyde resin prepolymer solution was 3 , and the optimal melamine-urea molar ratio was 0.4 .
\end{abstract}

Keywords: RDX/MUF; nanocomposite energetic microspheres; improved emulsion polymerization; MD simulation

\section{Introduction}

With the continuous development of technology and weapons systems, ammunition is required to ensure high accuracy, fierce power, and long range while maintaining high security in a variety of environments. High-energy and low-sensitivity ammunition is desired in the process of continuous improvement of the weapon system. Since cyclotrimethylene trinitramine has large crystal defects, it cannot meet the requirements of current weapon system development. Refined cyclotrimethylene trinitramine (RDX) has the characteristics of stable performance and high energy density, which can be applied to land, sea, and air force weapons and used as an energy source for explosive devices. However, 
there are major safety hazards, owing to its high mechanical sensitivity in storage, transportation and use procedures [1,2]. Desensitization coating of RDX can not only fairly well reduce the sensitivity of propellant, but also effectively avoid the phenomenon of "dehumidification" [3], thereby improving the safety performance of RDX. Domestic and foreign scholars have done more research on desensitization coating of RDX. Liu Jie et al. [4] prepared nano RDX-based polymer-bonded explosives (PBXs) by water suspension coating technology and analyzed their thermal decomposition properties. However, for the conventional water suspension coating, the desensitization effect was not ideal because of poor coating effect and severe particle leakage. Yu Chen et al. [5] obtained two RDX-based PBXs by studying the effect of recrystallization solvent on the morphology of RDX crystals, discovering high-quality RDX was critical to decreasing the sensitivity of PBX. Inspired by the report, we have prepared submicron RDX as the main explosive for the synthesis of PBX in this paper by using a novel green refinement technique. Shi Xiaofeng et al. [6] fabricated HP-RDX particles by spray drying technology, and the effect of insensitivity was obvious. It can be seen that effective coating of explosives is one of the commonly used measures to reduce sensitivity. The addition of desensitizing agent can effectively decrease the stress concentration of the explosive caused by external stimuli, thereby reducing the number of hot spots formed and achieving relative security.

In recent years, it has been a research hotspot for researchers at home and abroad to desensitize explosives by selecting adhesives with excellent performance or seeking new desensitization means. Choosing a suitable binder is essential to reducing the sensitivity of the explosive [7-10]. Thermosetting adhesive, urea-formaldehyde resin binder (UF), is one of the most demanding binders in the market. It is widely used in many fields, thanks to its rich raw material resources, simple manufacturing process, high initial viscosity, high bonding strength and low cost. Scholars have gradually attached great importance to traditional "trialdehyde" adhesives (urea-formaldehyde resin adhesive, phenolic resin adhesive and melamine formaldehyde resin adhesive). Not only can melamine-urea-formaldehyde (MUF) resin improve the storage performance in humid environments through co-polycondensation, but also the modified urea formaldehyde resin has a low free formaldehyde content, which can satisfy the requirements of green environmental protection and is considered to be the most promising polycondensation resin [11]. With the continuous improvement of the comprehensive performance of binders, reports on their applications are beginning to appear in the field of energetic materials. In 2015, Yang Zhijian et al. [12] used 3\% melamine formaldehyde resin monomer as the coating agent to coat CL-20, HMX and RDX, and successfully formed a dense and uniform melamine-formaldehyde (MF) resin coating on the surface of the coated particles, which greatly improved thermal stability and safety performance of the explosives. However, the shortcoming is that the synthetic factors of the coated composite particles were not discussed in the article, and their thermal properties were not analyzed either. This is actually quite an important subject, because the composite particles with the superior morphology and the best performance can be obtained under the optimal synthesis conditions. In practical terms, as a viscous heat-sensitive resin, MUF can not only offer protection to explosives on the surface of the RDX, but also improve the thermal stability of the RDX availably.

The compatibility between the binder and the explosive means the ability to maintain physical, chemical and explosive properties that do not change significantly after mixing both or bringing them into contact. It is an important property for high energy materials. If the explosive is incompatible with other components, the stability and explosion point will decrease, deteriorating the overall performance. There are many experimental methods for compatibility, but there are few works on how to determine these in theory. Binding energy can be used as a measure of the interaction force between the binder and the main explosive in the PBX system. And the binding energy is numerically a negative value of the interaction energy. The greater the binding energy is, the more stable the PBX system will be. That is, the compatibility between the main explosive and the binder is better exhibited [13]. Moreover, the differential scanning calorimetry (DSC) method is a commonly used approach for judging the compatibility level of components, taking the difference $\Delta \mathrm{Tp}$ between the decomposition peak temperature Tp of the mixed DSC and the decomposition peak temperature $T_{p 0}$ 
of the elemental explosive's DSC as a standard. The recommended rating for evaluating compatibility is in accordance with the $\mathrm{ABCD}$ criteria: Grade $\mathrm{A}, 0^{\circ} \mathrm{C} \sim 2^{\circ} \mathrm{C}$, the mixed system is in a compatible state; $B$ grade, $3{ }^{\circ} \mathrm{C} \sim 5^{\circ} \mathrm{C}$, the mixied system is slightly sensitive; $\mathrm{C}$ grade, $6{ }^{\circ} \mathrm{C} \sim 15^{\circ} \mathrm{C}$, the mixed system is sensitive; Class $\mathrm{D},>15^{\circ} \mathrm{C}$, the mixed system is in hazard. Therefore, in order to clarify the practical application value of RDX/MUF, we use the combination of molecular dynamics (MD) simulation and DSC to identify the compatibility of RDX and UF.

This report combined molecular dynamics simulation with experiments. First, MD was adopted to simulate the binding energy between RDX and UF, measuring the compatibility between RDX and UF. Afterwards, the simulation predicted the optimal reaction temperature for the preparation of RDX/MUF. The simulation results of the two parts are in line with the experimental results. Then, the improved emulsion polymerization method was applied to the RDX coating, and the process of fabricating RDX/MUF composite energetic microspheres was discussed for the first time. The method improved the general problem of irregular coating morphology and low mechanical strength of the coating layer in existing coating desensitization technology. Moreover, we explored the effects of different ratios of melamine $(\mathrm{M})$-urea $(\mathrm{U})$, precursor solution $\mathrm{pH}$, and reaction temperature on the morphology of RDX/MUF nano-energetic microspheres, and finally determined the optimal process conditions for the preparation of RDX/MUF nano-energetic microspheres via emulsion polymerization. The results have provided reliable references for broadening the scope of application of nitramine explosives and solving the contradiction between high energy output and good safety of explosives.

\section{Experiment Parts}

\subsection{Molecular Dynamics (MD) Simulation}

Aiming to determine the compatibility level between RDX and UF, and explore the optimal combination temperature of RDX and UF, MD simulation was carried out with the Compass force field under the Discover module, and the binding energy between RDX and UF is analyzed, taking the binding energy between the insensitive coating adhesive $\mathrm{F}_{2602}$ commonly used in the simulation and RDX as a reference. Meanwhile, DSC was conducted to study the compatibility level according to the ABCD criterion. Finally, the binding capacity between UF and RDX under $50{ }^{\circ} \mathrm{C}, 60^{\circ} \mathrm{C}, 70{ }^{\circ} \mathrm{C}$, $80^{\circ} \mathrm{C}, 90^{\circ} \mathrm{C}$ environments was simulated respectively, and the possible optimal reaction temperature was obtained.

The 6000-step set optimization on RDX cells was performed under the Forcite module, and then the $2 \times 2 \times 3$ RDX supercells were respectively established (the distribution of the cut pattern on the crystal face is shown in Figure 1a). The molecular structure of two polymer binders $F_{2602}$ and UF was drawn by Materials Studio (Figure $1 b, c$ ). Molecular mechanics optimization of $F_{2602}$ and UF was carried out to minimize the energy, and the optimized results were subjected to MD simulation. The constructed UF molecular chain was built into the vacuum layer of the RDX supercell, and the supercell space was compressed so that the model density is as close as possible to the theoretical density of each elemental explosive. Subsequently, energy minimization was processed on the two PBX models (Figure 1d,e), and simulations were performed according to the simulation parameters shown in Table 1.

Table 1. Simulation parameters of molecular dynamics.

\begin{tabular}{cccc}
\hline Parameters & Value & Parameters & Value \\
\hline Ensemble & Network Virtual Terminal & Force field & COMPASS \\
Temperature & $298 \mathrm{~K}$ & Thermostat & Andersen \\
Time & $300 \mathrm{ps}$ & Time step & $1 \mathrm{fs}$ \\
Frame output every & 5000 steps & Cut-off distance & $15.5 \AA$ \\
Spline width & $1 \AA$ & Buffer width & $0.5 \AA$ \\
\hline
\end{tabular}




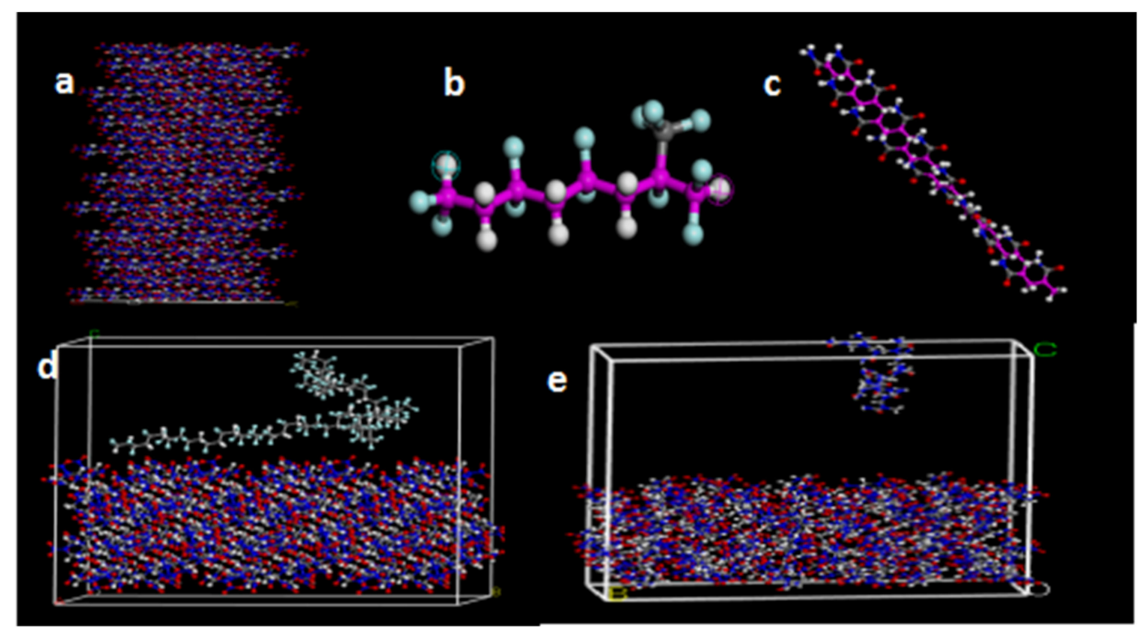

Figure 1. Structural model of different samples: (a) cross section of cyclotrimethylene trinitramine (RDX) unit cell; (b) $\mathrm{F}_{2602}$; (c) urea-formaldehyde resin binder (UF); (d) RDX/F 2602 ; (e) RDX/UF.

\subsection{Materials}

RDX was provided by Gansu Yinguang Chemical Industry Group Co, Baiyin, China. When preparing RDX/MUF composites by physical mixing and interfacial polymerization methods, the raw materials were recrystallized according to the reference [14]. Dimethyl sulfoxide was obtained from Tianjin Fuchen Chemical Reagent Factory, Tianjin, China. Tween 80 and Span80 were mixed as the composite emulsifier for explosives with $\mathrm{M}_{\text {Tween 80 }}: \mathrm{M}_{\text {Span80 }}$ of 0.57:0.43. Triethanolamine (TEOA, used to adjust the $\mathrm{pH}$ value during the reaction) was from Tianjin Sailboat Chemical Reagent Technology Co., Ltd, Tianjin, China. Urea, formaldehyde, hydrochloric acid (5\% dilute hydrochloric acid was used to adjust the $\mathrm{pH}$ value in the present study), and resorcinol (R-80) were provided by Tianjin Tianli Chemical Reagent Co., Ltd. Tianjin, China. Ammonium chloride was purchased from Tianjin Guangfu Technology Development Co., Ltd. Tianjin, China. Polyvinyl alcohol 2488 (PVA) was supplied by Qingdao Yousuo Chemical Technology Co., Ltd. Qingdao, China. Pure water was obtained from pure water supply of Taiyuan Iron and Steel Co., Ltd. Taiyuan, China.

\subsection{Synthesis}

RDX/MUF composite energetic microspheres were fabricated via improved emulsion polymerization. According to the three key steps of "preparation of RDX emulsion" $\rightarrow$ "synthesis and modification of UF prepolymer" $\rightarrow$ "spheroidization of RDX/MUF", the detailed preparation process is as follows. (1) Preparation of RDX emulsion. $6 \mathrm{~g}$ of RDX was added to $35 \mathrm{~mL}$ of deionized water, and $0.01 \mathrm{~g}$ of span- 80 was dropwise added as an emulsifier. The mixture was emulsified and sheared at a rate of $7000 \mathrm{rad} / \mathrm{min}$ for $30 \mathrm{~min}$ until a stable explosive emulsion was formed. (2) Synthesis and modification of UF prepolymer. $0.62 \mathrm{~g}$ of urea and $1.87 \mathrm{~g}$ of formaldehyde solution (the concentration is $37 \%$ ) were mixed, and then the urea was sufficiently dissolved with a magnetic stirrer. The $\mathrm{pH}$ of the solution was adjusted to 8.5 9.5 with triethanolamine. Then the system was placed in a water bath at $65^{\circ} \mathrm{C}$ for $1 \mathrm{~h}$ to prepare a transparent and viscous urea-formaldehyde resin prepolymer. After cooling, $\mathrm{HCl}$ was added dropwise until the $\mathrm{PH}$ value was tuned to about 3.5. Set aside. (3) Spheroidization of RDX/MUF. A certain quality of the RDX emulsion prepared in step (1) was taken out, and PVA at a concentration of $8 \%$, melamine, $0.125 \mathrm{~g}$ of resorcinol, $0.06 \mathrm{~g}$ of ammonium chloride were added in sequence. The $\mathrm{pH}$ value was adjusted to about 3.5 with dilute hydrochloric acid. Subsequently, a three-necked flask was placed in the water bath and reacted at $65^{\circ} \mathrm{C}$ for 3 to $4 \mathrm{~h}$, and then allowed to stand. After natural cooling, followed by vacuum filtration, deionized water washing and drying, ultimately high-quality RDX/MUF composite energetic microspheres were given, labeled as RDX/MUF-1. 
In order to compare and highlight the advantages of the improved emulsion polymerization process, RDX/MUF composite particles with the same MUF content were prepared by simple physical mixing, labeled as RDX/MUF-2. The schematic diagram of the preparation of RDX/MUF composite particles is displayed in Figure 2.

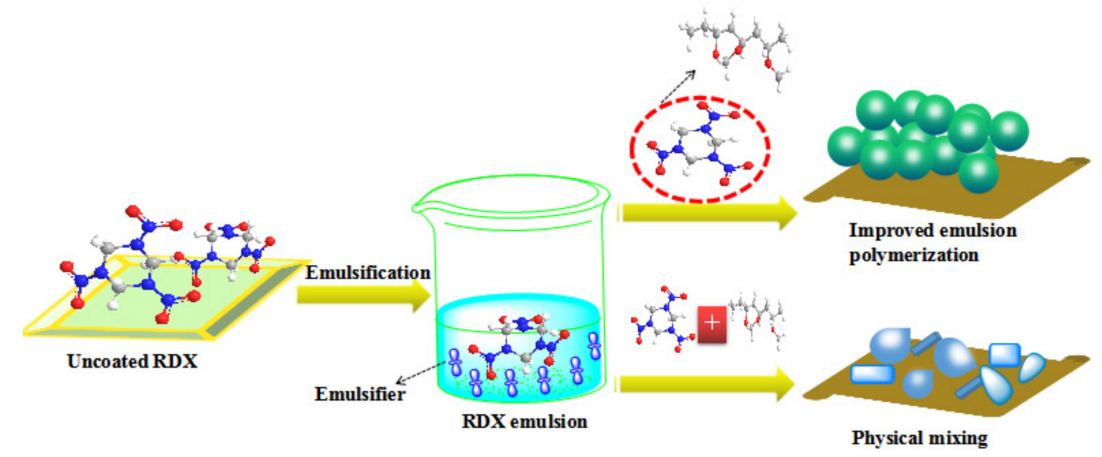

Figure 2. Schematic diagram of preparing RDX/melamine-urea-formaldehyde (MUF) composite particles.

\subsection{The Influence of Key Factors on the Morphology of Refined Cyclotrimethylene Trinitramine/Melamine-Urea-Formaldehyde (RDX/MUF)}

The effects of reaction temperature $\left(50^{\circ} \mathrm{C}, 60^{\circ} \mathrm{C}, 70^{\circ} \mathrm{C}, 80^{\circ} \mathrm{C}, 90^{\circ} \mathrm{C}\right)$, urea-formaldehyde resin prepolymer $\mathrm{pH}$ ( $\mathrm{pH}$ value was 1, 3, 5, 7, 9), and melamine-urea molar ratio (nM:nU $=0.2,0.3,0.4,0.5$, 0.6) on morphology and crystal structure of RDX/MUF composite energetic microspheres prepared via improved emulsion polymerization were studied by single factor test. The results are depicted in Figure 3.

As can be seen from Figure 3a-e, when the reaction temperature was $50{ }^{\circ} \mathrm{C}$ and $90{ }^{\circ} \mathrm{C}$, the morphology of the RDX/MUF composite particles was irregular. In the Figure 3a, the binder and RDX are bonded to each other. When filtered, it was found that white suspended particles were present in the filtrate. This is because the reaction temperature was too low, which went against the dissolution of melamine, and the white suspended particles in the filtrate were most likely the undissolved melamine. When the reaction temperature reached $90^{\circ} \mathrm{C}$, no white suspended particles appeared in the filtrate. However, it was apparent by scanning electron microscopy (SEM) that there were large particles in the composite particles and some of the RDX particles were exposed. This is attributed to the fact that excessive reaction temperature destroyed the regularity of the internal structure of the urea-formaldehyde resin, which caused the degree of crystallization of the urea-formaldehyde resin to decrease. In this case, the internal molecular arrangement became looser, the resin porosity increased, and the area occupied by the amorphous region also increased [15]. While the reaction temperature approached $60^{\circ} \mathrm{C}, 70^{\circ} \mathrm{C}$ and $80^{\circ} \mathrm{C}$, RDX particles can be better coated by MUF resin. SEM showed that although the composite energetic microspheres at $60^{\circ} \mathrm{C}$ and $80^{\circ} \mathrm{C}$ exhibited solid spherical structures, the particle size distribution were wide and some composite particles were bonded together. Whereas the RDX/MUF composite particles prepared at $70{ }^{\circ} \mathrm{C}$ were solid spheres with relatively uniform dispersion. Therefore, the optimal temperature for preparing RDX/MUF composite particles by emulsion polymerization was $70^{\circ} \mathrm{C}$, taking into account the environmental factors in the actual preparation of composite particles.

It can be seen from Figure $3 \mathrm{f}-\mathrm{j}$ that the morphology of RDX/MUF composite particles fabricated at different $\mathrm{pH}$ values of urea-formaldehyde resin prepolymer was quite different. When the $\mathrm{pH}$ of the urea-formaldehyde resin prepolymer was neutral and alkaline, the prepared polymer had very poor regularity and the particles were obviously exposed. This is because when the $\mathrm{pH}$ of the urea-formaldehyde resin prepolymer was 7 and 9, it did not reach the acidity required for the three-dimensional polycondensation of urea-formaldehyde resin, and the curing-effect was very poor, resulting in a large amount of amorphous regions. However, when the $\mathrm{pH}$ of the urea-formaldehyde resin prepolymer was acidic, the RDX/MUF composite particles exhibited a 
relatively regular morphology. But when the $\mathrm{pH}$ value was 5 , the composite particles showed a distinct "twinning" bond phenomenon. This is due to the fact that at this moment $-\mathrm{CH} 2 \mathrm{OH}$ in the reaction system mainly undergone more condensation reaction rather than participating in three-dimensional polycondensation [16]. The optimal $\mathrm{pH}$ of the urea-formaldehyde resin prepolymer for the preparation of RDX/MUF composite energetic microspheres by emulsion polymerization method can be considered to be 3 in combination with the SEM image of Figure $3 g$.

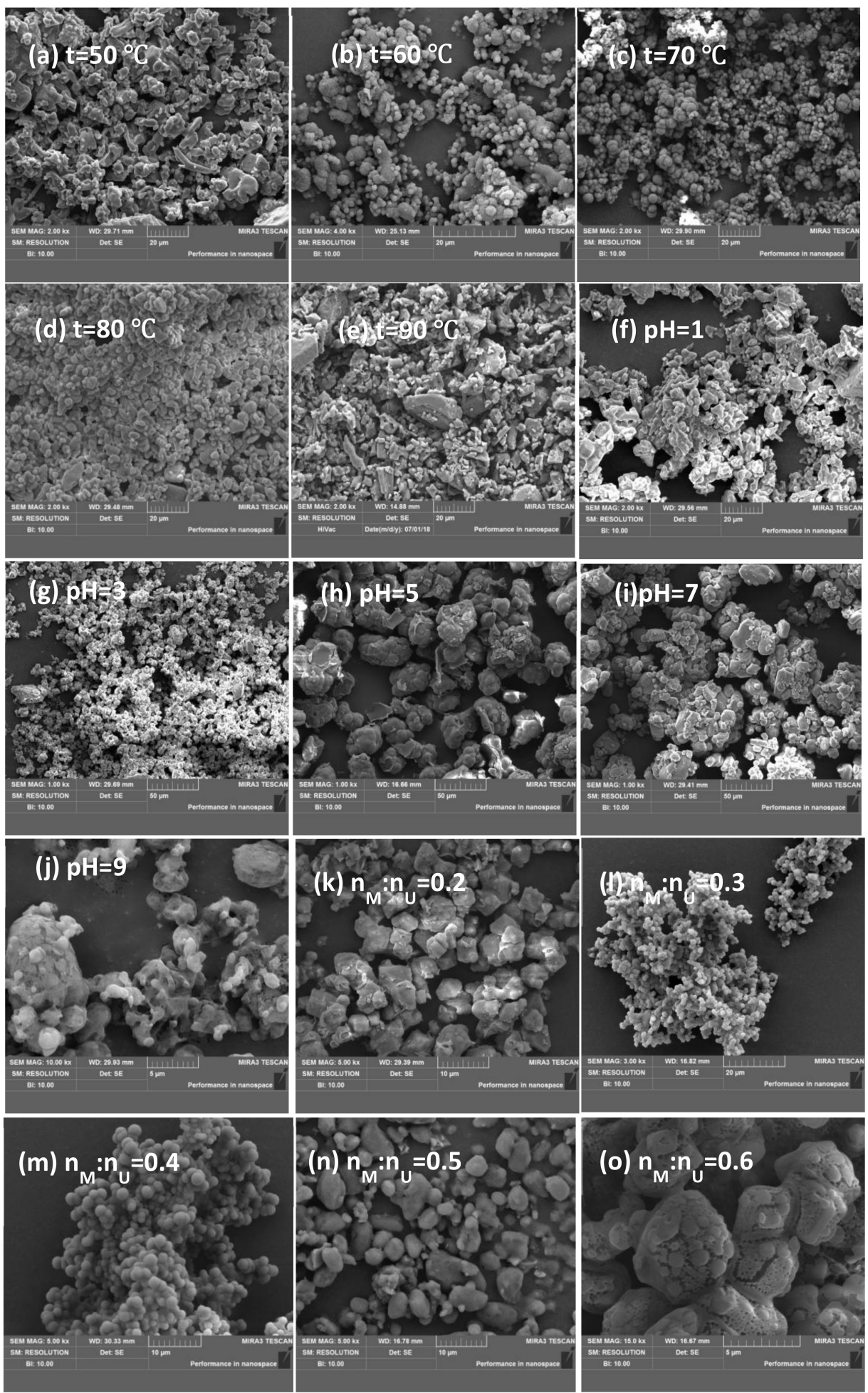

Figure 3. Scanning electron micrograph (SEM) of RDX/MUF composite energetic microspheres prepared under different conditions. 
As can be seen from Figure 3k-o, the morphology of RDX/MUF composite particles obtained under different melamine-urea ratios also showed big differences. When the melamine-urea ratio exceeded 0.4 , macromolecular chains appeared in the RDX/MUF composite particles, polymer molecules agglomerated and the molecular weight distribution was uneven. This can be explained by the fact that as the amount of melamine added increased, the formaldehyde content was relatively insufficient. At this point, some of the melamine could not participate in the reaction, leading to the deposition of white suspended particles onto the surface of the RDX/MUF composite particles. When the melamine-urea molar ratio was 0.4, the given RDX/MUF composite particles were spherical composite particles with smooth surface and uniform particle size distribution. This can be ascribed to the fact that melamine contains a triazine ring structure (involving 6 active groups), its reactivity is much greater than that of urea (containing 4 active groups), and it can produce more branched structures, which facilitates the copolymerization and crosslinking of urea-formaldehyde resin system to form composite particles with excellent morphology. However, when the melamine content was low, the curing speed of the urea-formaldehyde resin was reduced, causing mutual adhesion [17]. That is, the optimal melamine-urea molar ratio for fabricating RDX/MUF composite energetic microspheres by emulsion polymerization was 0.4 .

All in all, RDX/MUF composite energetic microspheres were prepared via emulsion polymerization under the following conditions: where the reaction temperature was $70{ }^{\circ} \mathrm{C}$, the urea-formaldehyde resin prepolymer solution had a $\mathrm{pH}$ of 3 , the $\mathrm{n}(\mathrm{M}) \mathrm{n}(\mathrm{U})$ ratio was 0.4 , and $\mathrm{RDX}$ aqueous suspension was emulsified at $7000 \mathrm{rad} / \mathrm{min}$ for $30 \mathrm{~min}$. Subsequently, the resultant RDX/MUF composite energetic microspheres were analyzed.

\section{Results and Discussion}

\subsection{Stability Analysis of RDX/Urea-Formaldehyde Resin Binder (UF)}

The stability of RDX/UF was calculated and analyzed by DSC method and MD simulation. The results are shown in Figure 4:
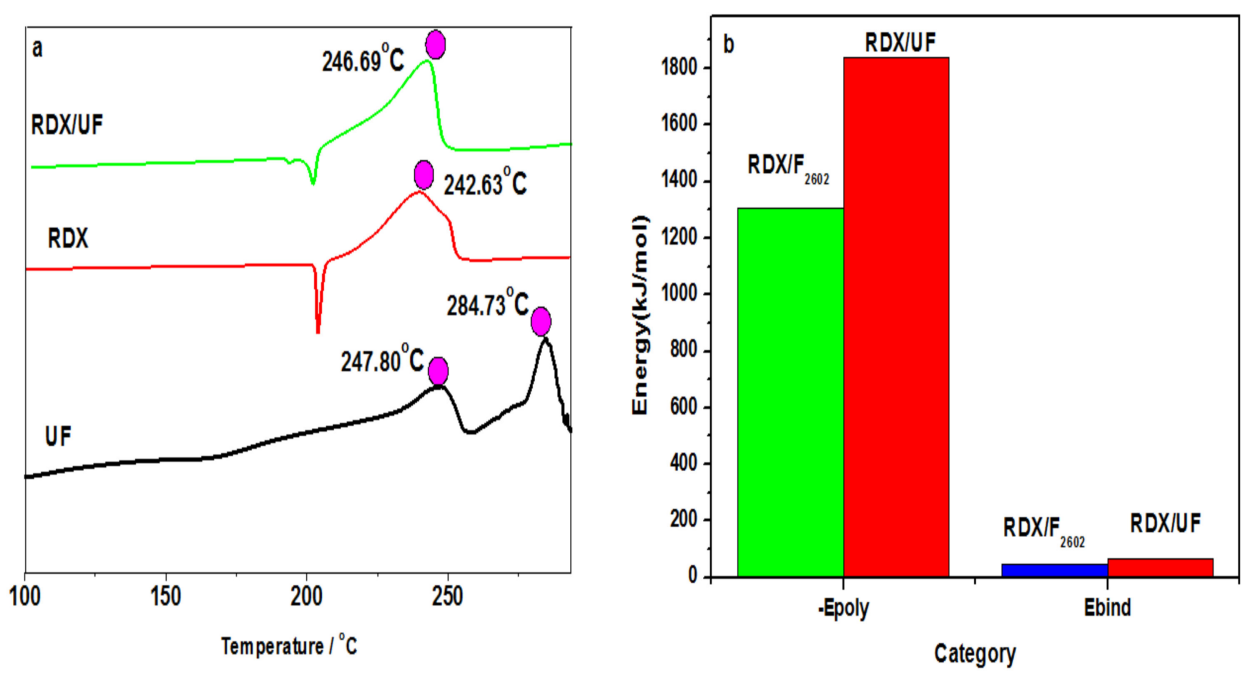

Figure 4. Results of stability analysis: (a) differential scanning calorimetry (DSC) method and (b) molecular dynamics (MD) simulation.

Figure 4a shows that there is a single exothermic peak in the DSC curve of RDX. The difference $\Delta \mathrm{Tp}$ between the decomposition peak temperature of RDX/UF and that of RDX is $4.06{ }^{\circ} \mathrm{C}$. According to the ABCD criteria, the compatibility level of RDX/UF is B grade, indicating that RDX/UF exhibits good thermal stability. Importantly, it can be seen from the MD simulation results in Figure $4 \mathrm{~b}$ that 
the binding energy of RDX/UF is greater than that of RDX/F2602, that is, there is a strong interaction between UF and RDX, which has better stability.

\subsection{Molecular Dynamics (MD) Simulation of Optimal Reaction Temperature}

In order to explore the interaction force between UF and RDX under different temperature systems, the binding energy of RDX/MUF was simulated by $\mathrm{MD}$ at $50{ }^{\circ} \mathrm{C}, 60{ }^{\circ} \mathrm{C}, 70{ }^{\circ} \mathrm{C}, 80{ }^{\circ} \mathrm{C}$ and $90{ }^{\circ} \mathrm{C}$, respectively. And the simulation results are shown in Figure 5:

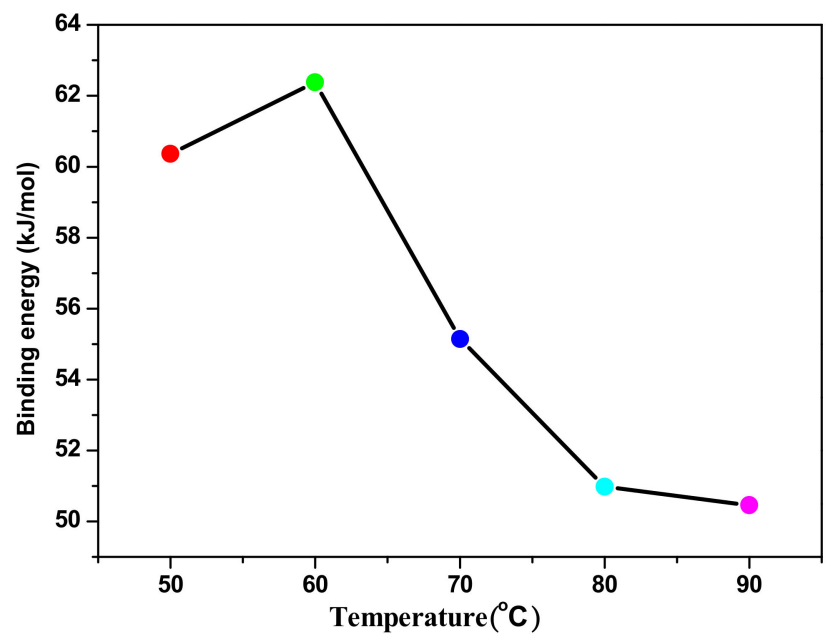

Figure 5. The line chart for MD simulation of the binding energy between UF and RDX under different temperature systems.

It can be seen from Figure 5 that the interface binding energy of RDX and UF increases first and then decreases continuously with the increase of temperature, and the optimal combination temperature can be predicted to be between $60^{\circ} \mathrm{C}$ and $70^{\circ} \mathrm{C}$. This is because the total energy of the RDX/UF system raises as the temperature increases. The increasing spacing between RDX and UF leads to weaker interactions between molecules. The weakening effect between molecules is greater than the increasing effect of total energy, resulting in a decrease in binding energy $[18,19]$.

\subsection{Morphologies of RDX before and after Coating}

Figure 6 illustrates the morphology of different RDX samples. From Figure 6a, we can see that the surface of the raw RDX crystal has obvious defects and the particle size distribution is uneven. For the refined RDX, the particle size becomes significantly smaller, it has a narrow particle size distribution, and the crystal morphology is smooth and tends to be spherical, as shown in Figure $6 \mathrm{~b}$. Under the condition that the reaction temperature was $70{ }^{\circ} \mathrm{C}$, the $\mathrm{pH}$ of the urea-formaldehyde resin prepolymer solution was 3, and the $\mathrm{n}(\mathrm{M}) \mathrm{n}(\mathrm{U})$ was 0.4 , RDX/MUF particles prepared by emulsion polymerization were solid spheres with smooth surface and uniform dispersion, as shown in Figure 6c. The particle size of the RDX/MUF-1 composite microspheres is larger than that of the refined RDX, but the particle size distribution range is still narrow, which may be caused by the formation of a uniform MUF protective layer on the surface of the refined RDX. It is noticeable that the composite particles obtained by the improved emulsion polymerization method have a dense surface without any particle exposure problems, while for RDX/MUF prepared by simple physical mixing method, there exist obviously exposed particles (Figure 6d). This is because on the one hand, during the preparation of RDX/MUF-1 the dispersant is oriented and adsorbed on the surface of RDX, which improves the wettability of the surface of RDX and prevents the RDX particles from agglomerating each other, so that the particle size distribution of the as-prepared particles is relatively uniform. On the other hand, the spheroidization process of RDX/MUF-1 is accomplished by the spontaneous addition reaction and polycondensation reaction of molecules freed on the surface of RDX, remarkably enhancing the final spheroidization 
effect. However, the forming process of RDX/MUF-2 mainly depends on the combination of hydrogen bonding and mechanical force between RDX molecules and MUF under the action of mechanical agitation. Therefore, the coating coverage of RDX/MUF-2 particles, with severe RDX leakage, is not high, presenting poor coating effect.

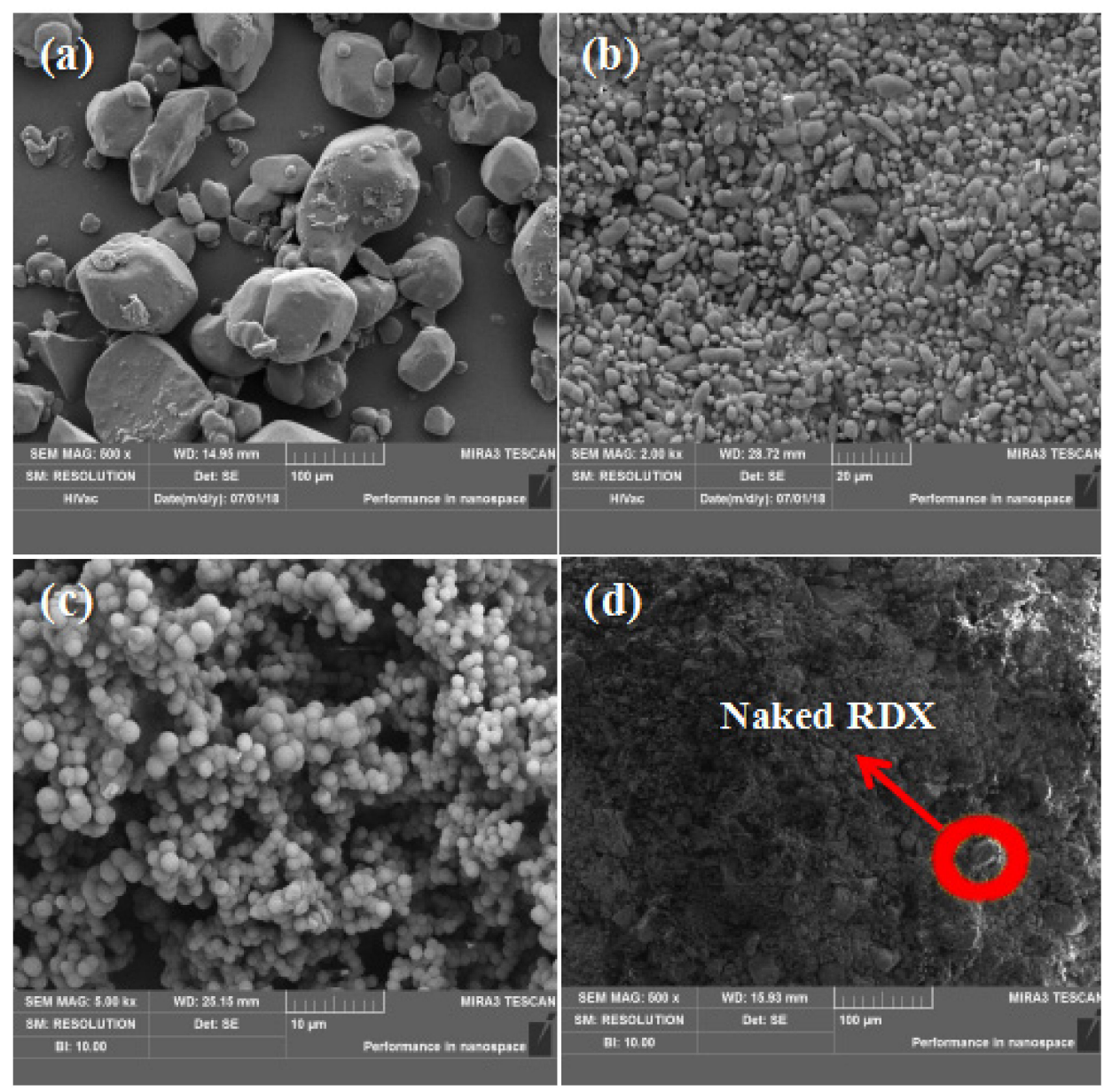

Figure 6. SEM of different RDX samples: (a) raw RDX, (b) refined RDX, (c) RDX/MUF-1, (d) RDX/MUF-2.

\subsection{Thermal Properties}

In order to explore the difference in thermal decomposition properties between RDX/MUF composite particles prepared by different methods, the DSC test was performed on the RDX samples before and after coating with a DSC131 differential scanning calorimeter at the heating rate of 5, 10, 15, $20{ }^{\circ} \mathrm{C} / \mathrm{min}$. The result is displayed in Figure 7 .

Figure 7 shows that the DSC curve of the RDX sample has a steep spike, and the heat release rate is very fast. This is attributed to the fact that in the molecular structure of RDX, the three nitramine groups contain two upright bonds and one flat bond. The melting process is greatly affected by the induction effect of the substituent, and RDX is homogeneously decomposed in the molten state after the completion of melting. The acceleration of the reaction resulting from the simultaneous phase change during this decomposition is the main cause of the steep peak of the DSC curve of RDX.

It can be observed that the raw RDX (Figure 7a), Refined RDX (Figure 7b), RDX/MUF-1 (Figure 7c), and RDX/MUF-2 (Figure 7d) composite energetic microspheres have similar thermal decomposition behaviors, possessing a very distinct endothermic peak at around $204{ }^{\circ} \mathrm{C}$. That is, the addition of the MUF binder has little influence on the stability of RDX crystal form. 

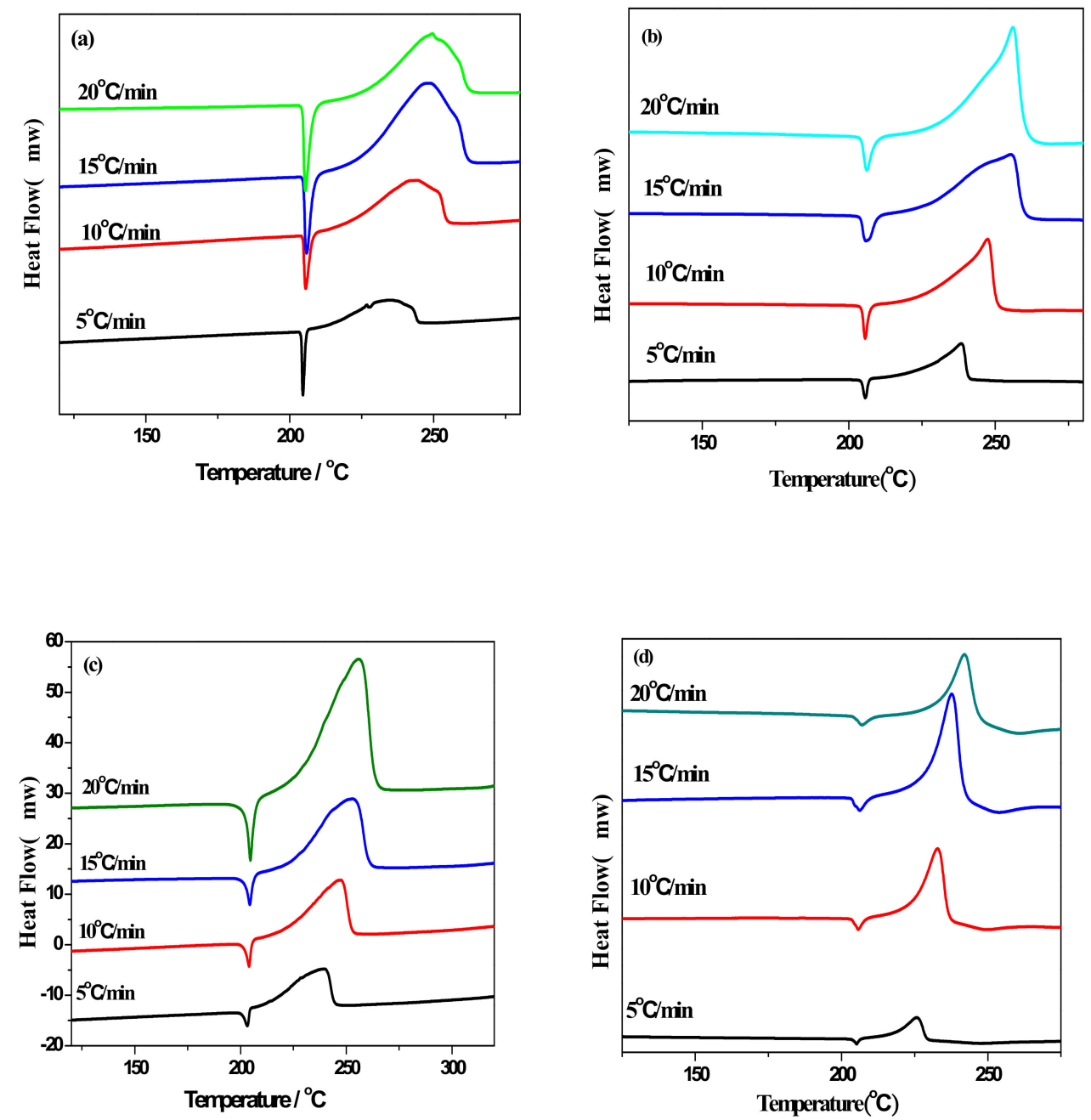

Figure 7. DSC curves of RDX particles: (a) Raw RDX; (b) Refined RDX; (c) RDX/MU-1; (d) RDX/MUF-2.

Based on the decomposition peak temperature values of three different RDX samples at different heating rates, in order to ensure the accuracy of the calculation the decomposition activation energy $E_{a}$ and the pre-exponential factor A of the four RDX samples were calculated by the Kissinger formula, Ozawa formula and Starink formula, respectively [20,21].

$$
\begin{gathered}
\ln \left(\frac{\beta}{\mathrm{T}_{\mathrm{p}}^{2}}\right)=\ln \left(\frac{\mathrm{AR}}{E_{\mathrm{a}}}\right)-\frac{E_{\mathrm{a}}}{\mathrm{RT}_{\mathrm{p}}} \\
\lg \beta=\lg \left(\frac{A E_{a}}{R G(a)}\right)-2.315-0.4567 \frac{E_{a}}{R T} \\
\ln \left(\frac{\beta}{T^{1.8}}\right)=C s-1.0037 \frac{E}{R T}
\end{gathered}
$$

In the Formulas (1)-(3): $\mathrm{Tp}$ is the decomposition peak temperature value of the explosive at the heating rate $\beta, \mathrm{K} ; R$ is the gas constant, $8.314 \mathrm{~J} \cdot \mathrm{mol}^{-1} \cdot \mathrm{K}^{-1} ; G_{(a)}$ is the integral form of the reaction mechanism function; $\beta$ is the heating rate, $\mathrm{K} \cdot \mathrm{min}^{-1} ; A$ is the pre-factor, $\mathrm{min}^{-1} \mathrm{or} \mathrm{s}^{-1} ; E_{a}$ is the apparent activation energy, $\mathrm{kJ} \cdot \mathrm{mol}^{-1} ; C_{s}$ is a constant.

The apparent activation energy $\left(E_{a}\right)$ acquired through the Kissinger formula and the peak temperature $\left(T_{p 0}\right)$ obtained by Formula (4) when the heating rate $\beta$ tends to zero were brought into Formula (5) to figure out the thermal explosion critical temperature $T_{b}$ [22]. 


$$
\begin{gathered}
T_{p i}=T_{p 0}+b \beta_{i}+c \beta_{i}{ }^{2}+d \beta_{i}{ }^{3} \\
T_{b}=\frac{E_{a}-\sqrt{\left(E_{a}{ }^{2}-4 R E_{a} T_{p 0}\right)}}{2 R}
\end{gathered}
$$

The calculation results are listed in Table 2:

Table 2. Thermal decomposition kinetic parameters of different RDX samples.

\begin{tabular}{ccccccccc}
\hline \multirow{2}{*}{ Samples } & \multicolumn{4}{c}{ Kinetic Factor } & \multicolumn{3}{c}{ Thermal Stability } \\
\cline { 2 - 9 } & $\boldsymbol{E}_{\boldsymbol{a} \mathbf{1}}$ & $\boldsymbol{E}_{\boldsymbol{a} \mathbf{2}}$ & $\boldsymbol{E}_{\boldsymbol{a} \mathbf{3}}$ & $\boldsymbol{E}_{\boldsymbol{a}}$ & $\mathbf{l g} \mathbf{A}$ & $\mathbf{R}$ & $\boldsymbol{T}_{\mathbf{p} \mathbf{0}} /{ }^{\circ} \mathbf{C}$ & $\boldsymbol{T}_{\mathbf{b}} /{ }^{\circ} \mathbf{C}$ \\
\hline Raw RDX & 176.23 & 184.83 & 177.09 & 179.38 & 14.65 & 0.98 & 220.58 & 231.04 \\
Refined RDX & 163.53 & 172.18 & 164.39 & 166.70 & 13.27 & 1 & 225.46 & 228.09 \\
RDX/MUF-1 & 205.88 & 186.16 & 178.37 & 190.13 & 14.78 & 0.99 & 228.52 & 231.02 \\
RDX/MUF-2 & 174.98 & 183.40 & 175.82 & 178.07 & 14.96 & 1 & 215.47 & 217.72 \\
\hline
\end{tabular}

In the table, $E_{\mathrm{a} 1}, E_{\mathrm{a} 2}$, and $E_{\mathrm{a} 3}$ are the activation energies obtained by the Kissinger formula, the Ozawa formula, and the Starink formula, respectively. $E_{\mathrm{a}}$ is the average of the three, with the unit for $\mathrm{kJ} / \mathrm{moL}$.

Compared with the raw RDX, the $\mathrm{E}_{\mathrm{a}}$ of the refined RDX decreased from $179.38 \mathrm{~kJ} / \mathrm{moL}$ to $166.70 \mathrm{~kJ} / \mathrm{moL}$, the $\mathrm{Tb}$ decreased from $231.04{ }^{\circ} \mathrm{C}$ to $228.09^{\circ} \mathrm{C}$, and the thermal stability was weakened. On the one hand, this is caused by the strong surface effect, quantum size effect and macroscopic quantum tunneling effect of the particles after the particle size is reduced. On the other hand, as the particle size of RDX becomes smaller, the surface area gets larger, so that the external energy absorbed over a period of time increases. The heated area and the reactivity are correspondingly improved, so $E_{\mathrm{a}}$ and $T_{\mathrm{b}}$ are reduced in comparison with the raw RDX. Interestingly, after adding MUF binder, the increment of the $E_{\mathrm{a}}$ of RDX/MUF- 1 and RDX/MUF- 2 was $22.43 \mathrm{~kJ} / \mathrm{moL}$ and $11.37 \mathrm{~kJ} / \mathrm{moL}$, respectively, as compared with the refined RDX. It is visible that MUF can effectively improve the thermal decomposition performance of RDX. Notably, the $T_{b}$ of RDX/MUF- 1 is increased by about $3{ }^{\circ} \mathrm{C}$, while the $T_{\mathrm{b}}$ of RDX/MUF-2 is almost unchanged, which may be related to the coating morphology and compactness of RDX/MUF. The composite particles obtained by the improved emulsion polymerization method have a substantially dense and uniform coating layer with high coverage, and no particles are exposed, which are beneficial to improve the stability of the thermal decomposition process of RDX/MUF composite particles. The uniform and excellent morphology of the composite particles will be conducive to the improvement of the thermal stability of the composite particles. Therefore, aiming to improve the thermal stability of such composite particles, it is preferred to select the emulsion polymerization method to prepare the composite particles with good morphology, which may be a favorable way to reduce heat loss.

\subsection{Safety Performance}

According to the experimental method of the national military standard GJB772A-97, the impact sensitivity and friction sensitivity of the virgin RDX and different RDX-based composite particles were tested. The test results are shown in Figure 8a,b.

It can be seen from Figure 8 that MUF has a remarkable effect on RDX, among them, RDX/MUF-1 fabricated by the improved emulsion polymerization method has the best desensitization effect. Compared with the raw RDX, the $\mathrm{H}_{50}$ of the refined RDX, RDX/MUF- 1 and RDX/MUF-2 increased from $29.32 \mathrm{~cm}$ to $44.5 \mathrm{~cm}, 82.4 \mathrm{~cm}$, and $60.7 \mathrm{~cm}$, respectively. The probability of frictional explosion decreased from $96 \%$ to $92 \%, 8 \%$, and $60 \%$, respectively, showing an ideal desensitization effect with security performance efficiently improved. This can be explained by hotspot theory [23]. The surface of the refined RDX particles tends to be smooth, and the internal defects gradually decrease, which increases 
the heat transfer rate between RDX particles, and hinders the formation of hot spots when encountering external stimuli, thus the security performance is improved. Similarly, since the MUF binder is successfully coated on the surface of RDX, it can generate a certain buffering effect under external mechanical stimulation, effectively slowing down the formation of hot spots. Clearly, the safety performance of RDX/MUF-1 and RDX/MUF-2 varies greatly with the same binder component. This is ascribed to the fact that the RDX/MUF composite particles prepared by the improved emulsion polymerization method are more uniform in each particle. The small and uniform particle size distribution between the particles will give rise to the increase of the gap between the particles. As a consequence, for the same quality RDX particles, the pressed area is increased, which reduces the stress concentration between the particles and prevents the generation of local hotspots.
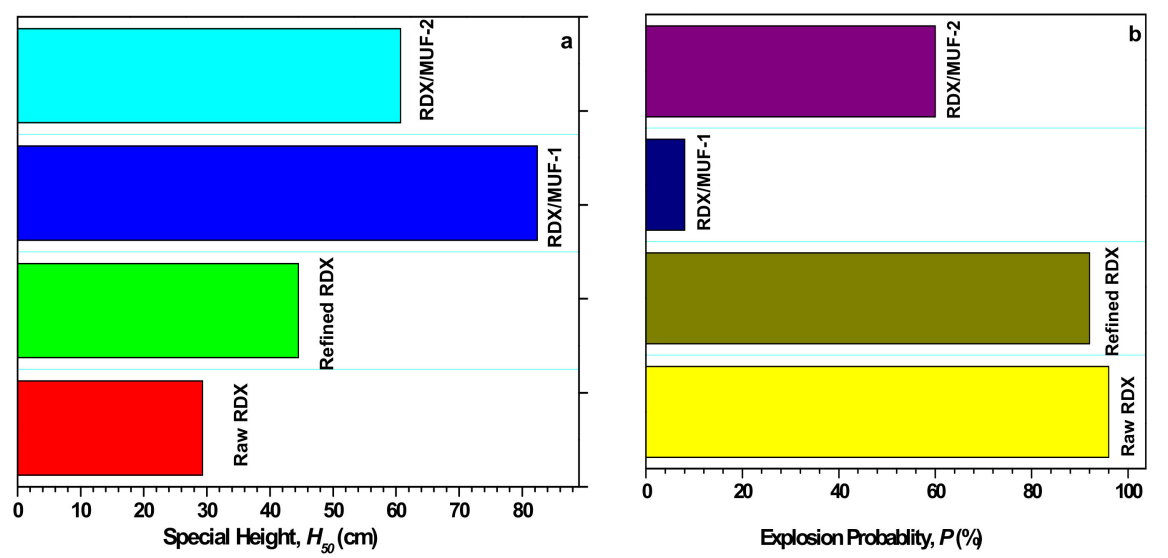

Figure 8. Sensitivity histograms for different RDX samples: (a) impact sensitivity, (b) friction sensitivity.

\section{Conclusions}

This report provides an improved emulsion polymerization technique for the fabrication of insensitive RDX/MUF composite particles with outstanding spherical effects and uniform particle size distribution. Based on this, such a novel approach can improve mechanical strength and optimize the coating morphology. In the meantime, the combination of MD simulation and the DSC method shows that RDX/UF has good stability, and the comprehensive performance of RDX after coating is not significantly deteriorated. We focused on the influences of different ratios of melamine-urea, precursor solution $\mathrm{pH}$, and reaction temperature on the morphology of RDX/MUF nano-energetic microspheres. The $\mathrm{H}_{50}$ of the resultant RDX/MUF composites prepared under these conditions, compared with the uncoated RDX, increased from $44.5 \mathrm{~cm}$ to $82.4 \mathrm{~cm}$, and the frictional explosion probability decreased from $92 \%$ to $8 \%$, thereby significantly enhancing the safety performance; $E_{\mathrm{a}}$ increased from $166.70 \mathrm{~kJ} / \mathrm{mol}$ to $190.13 \mathrm{~kJ} / \mathrm{mol}$, and $T_{\mathrm{p}}$ increased from $228.09{ }^{\circ} \mathrm{C}$ to $231.02{ }^{\circ} \mathrm{C}$, slightly improving the thermal stability. This study can present an important reference for settling the contradiction between high energy output and good safety for explosives and broadening the application range of nitramine explosives.

Author Contributions: X.J. designed the experiments and wrote the paper; Y.H., L.X., X.L., Y.M., performed the experiments; J.W., M.F. and J.X. checked the paper. All authors read and approved the manuscript. Everyone contributed in reviewing and enriching the content.

Funding: This research received no external funding.

Conflicts of Interest: The authors declare no conflict of interest.

\section{References}

1. Kumar, R.; Siril, P.F.; Soni, P. Preparation of Nano-RDX by Evaporation Assisted Solvent-Antisolvent Interaction. Propellants Explos. Pyrotech. 2014, 39, 383-389. [CrossRef] 
2. He, B.; Stepanov, V.; Qiu, H.; Krasnoperov, L.N. Production and Characterization of Composite Nano-RDX by RESS Co-Precipitation. Propellants Explos. Pyrotech. 2015, 40, 659-664. [CrossRef]

3. Padfield, J.; Ferran, M.D.; Linmei, A.L. Chemical Desensitization of Explosives. Part 1. Effect of Ethyl Centralite on the Properties of an RDX-Based PBX. J. Energ. Mater. 2014, 32, 106-116. [CrossRef]

4. Liu, J.; Bao, X.Z.; Rong, Y.B.; Hao, G.Z.; Xiao, L.; Gao, H.; Ke, X.; Chen, T.; Jiang, W.; Li, F.S. Preparation of nano-RDX-based PBX and its thermal decomposition properties. J. Therm. Anal. Calorim. 2017, 131, 2693-2698. [CrossRef]

5. Wang, Y.; Li, X.; Chen, S.; Ma, X.; Yu, Z.; Jin, S.; Li, L.; Chen, Y. Preparation and Characterization of Cyclotrimethylenetrinitramine (RDX) with Reduced Sensitivity. Materials 2017, 10, 974. [CrossRef]

6. Shi, X.F.; Li, X.-D.; Wang, J.Y.; Ji, W. Preparation and thermal properties of RDX based ultrafine spherical coated particles. J. Solid Rocket Technol. 2015, 38, 383-386.

7. Wang, S.; An, C.; Wang, J.; Ye, B. Reduce the Sensitivity of CL-20 by Improving Thermal Conductivity Through Carbon Nanomaterials. Nanoscale Res. Lett. 2018, 13, 85-92. [CrossRef]

8. Hamedi, G.H. Effects of Polymeric Coating the Aggregate Surface on Reducing Moisture Sensitivity of Asphalt Mixtures. Int. J. Civ. Eng. 2017, 16, 1097-1107. [CrossRef]

9. Guo, L.; Yang, Z.; Li, Y.; Zu, B.; Dou, X. Sensitive, real-time and anti-interfering detection of nitro-explosive vapors realized by $\mathrm{ZnO} / \mathrm{rGO}$ core/shell micro-Schottky junction. Sens. Actuators B Chem. 2017, 239, $286-294$. [CrossRef]

10. Lindskog, C.; Asplund, A.; Engkvist, M.; Uhlen, M.; Korsgren, O.; Ponten, F. Antibody-based proteomics for discovery and exploration of proteins expressed in pancreatic islets. Discov. Med. 2010, 9, 565-578.

11. Gao, Q.; Shi, S.Q.; Zhang, S.; Li, J.; Wang, X.; Ding, W.; Liang, K.; Wang, J. Soybean meal-based adhesive enhanced by MUF resin. J. Appl. Polym. Sci. 2012, 125, 3676-3681. [CrossRef]

12. Yang, Z.; Ding, L.; Wu, P.; Liu, Y.; Nie, F.; Huang, F. Fabrication of RDX, HMX and CL-20 based microcapsules via in situ polymerization of melamine-formaldehyde resins with reduced sensitivity. Chem. Eng. J. 2015, 268, 60-66. [CrossRef]

13. Fu, Y.Z.; Liu, Y.Q.; Lan, Y.H. Molecular Dynamics Simulation on Compatibility of Hydroxyl-Terminated Polybutadiene/Plasticizer Blends. Acta Phys. Chim. Sin. 2009, 25, 1267-1272.

14. Jia, X.; Wang, J.; Hou, C.; Tan, Y. Green Preparation, SpHeroidal, and Superior Property of Nano-1, 3, 5, 7-Tetranittro-1, 3, 5, 7-Tetrazocane. J. Nanomater. 2018, 2018, 5839037. [CrossRef]

15. No, B.Y.; Kim, M.G. Syntheses and properties of low-level melamine-modified urea-melamine-formaldehyde resins. J. Appl. Polym. Sci. 2010, 93, 2559-2569. [CrossRef]

16. Cao, X.X.; Zhang, Y.H.; Gu, J.; Tan, H. Study on the Curing Characteristics and Synthesis Process of Modified Urea-formaldehyde Resin with Low Formaldehyde Release. China For. Prod. Ind. 2017, 44, 20-24.

17. Tohmura, S.; Inoue, A.; Sahari, S.H. Influence of the melamine content in melamine-urea-formaldehyde resins on formaldehyde emission and cured resin structure. J. Wood Sci. 2001, 47, 451-457. [CrossRef]

18. Lan, Y.; Zhai, J.; Li, D.; Yang, R. Multiscale Simulation on the Influence of Dimethyl Hydantoin on Mechanical Properties of GAP/RDX Propellants. Propellants Explos. Pyrotech. 2014, 39, 18-23. [CrossRef]

19. Xiao, J.; Fang, G.; Ji, G.; Xiao, H. Simulation investigations in the binding energy and mechanical properties of HMX-based polymer-bonded explosives. Chin. Sci. Bull. 2005, 50, 21-26. [CrossRef]

20. Hou, C.; Jia, X.; Wang, J. Efficient Preparation and Performance Characterization of the HMX/F2602 MicrospHeres by One-Step Granulation Process. J. Nanomater. 2017, 2017, 3607383. [CrossRef]

21. Wang, Y.; Song, X.; Song, D.; Liang, L.; An, C.; Wang, J. Synthesis, thermolysis, and sensitivities of HMX/NC energetic nanocomposites. J. Hazard. Mater. 2016, 312, 73-83. [CrossRef] [PubMed]

22. Xiao, J.; Huang, H.; Li, J.; Zhang, H.; Zhu, W.; Xiao, H. Computation of interface interactions and mechanical properties of HMX-based PBX with Estane 5703 from atomic simulation. J. Mater. Sci. 2008, 43, 5685-5691. [CrossRef]

23. Barua, A.; Kim, S.; Horie, Y.; Zhou, M. Ignition criterion for heterogeneous energetic materials based on hotspot size-temperature threshold. J. Appl. Phys. 2013, 113, 5794-5799. [CrossRef]

(C) 2019 by the authors. Licensee MDPI, Basel, Switzerland. This article is an open access article distributed under the terms and conditions of the Creative Commons Attribution (CC BY) license (http://creativecommons.org/licenses/by/4.0/). 